\title{
Parameters of diffusional kurtosis imaging for the diagnosis of acute cerebral infarction in different brain regions
}

\author{
YUE-LIN GUO ${ }^{1}$, SU-JUAN LI ${ }^{1}$, ZHONG-PING ZHANG ${ }^{2}$, ZHI-WEI SHEN ${ }^{1}$, GUI-SHAN ZHANG ${ }^{3}$, \\ GEN YAN $^{1}$, YAN-TING WANG ${ }^{1}$, HAI-BING RAO ${ }^{1}$, WEN-BIN ZHENG ${ }^{1}$ and REN-HUA WU ${ }^{1}$ \\ ${ }^{1}$ Department of Radiology, The Second Affiliated Hospital of Shantou University Medical College, \\ Shantou, Guangdong 515000; ${ }^{2}$ GE Healthcare, Guangzhou, Guangdong 510623; \\ ${ }^{3}$ College of Engineering, Shantou University, Shantou, Guangdong 515000, P.R. China
}

Received March 27, 2015; Accepted April 29, 2016

DOI: $10.3892 / \mathrm{etm} .2016 .3390$

\begin{abstract}
Diffusional kurtosis imaging (DKI) is a new type diffusion-weighted sequence which measures the non-Gaussianity of water diffusion. The present study aimed to investigate whether the parameters of DKI could distinguish between differences in water molecule diffusion in various brain regions under the conditions of acute infarction and to identify the optimal DKI parameter for locating ischemic lesions in each brain region. A total of 28 patients with acute ischemic stroke in different brain regions were recruited for the present study. The relative values of DKI parameters were selected as major assessment indices, and the homogeneity of background image and contrast of adjacent structures were used as minor assessment indices. According to the brain region involved in three DKI parametric maps, including mean kurtosis (MK), axial kurtosis $(\mathrm{Ka})$ and radial kurtosis $(\mathrm{Kr}), 112$ groups of regions of interest were outlined in the following regions: Corpus callosum $(n=17)$; corona radiata $(n=26)$; thalamus $(n=21)$; subcortical white matter $(n=24)$; and cerebral cortex $(n=24)$. For ischemic lesions in the corpus callosum and corona radiata, significant increases in relative $\mathrm{Ka}$ were detected, as compared with the other parameters $(\mathrm{P}<0.05)$. For ischemic lesions in the thalamus, subcortical white matter and cerebral cortices, an increase in the three parameters was detected, however this difference was not significant. Minor assessment indices demonstrated that Ka lacked tissue contrast and the background of $\mathrm{Kr}$ was heterogeneous; thus, MK was the
\end{abstract}

Correspondence to: Professor Ren-Hua Wu, Department of Radiology, The Second Affiliated Hospital of Shantou University Medical College, 69 Dong Xia Bei Road, Shantou, Guangdong 515000, P.R. China

E-mail: stkhuang@163.com

Abbreviations: DKI, diffusional kurtosis imaging; MK, mean kurtosis; Ka, axial kurtosis; Kr, radial kurtosis; rMK, relative mean kurtosis; rKa, relative axial kurtosis; rKr, relative radial kurtosis

Key words: ischemia, stroke, diffusion, kurtosis, magnetic resonance imaging superior assessment parameter for ischemic lesions in these regions. In conclusion, $\mathrm{Ka}$ is better suited for the diagnosis of acute ischemic lesions in highly anisotropic brain regions, such as the corpus callosum and corona radiate. MK may be appropriate for the lesions in low anisotropic or isotropic brain regions, such as the thalamus, subcortical white matter and cerebral cortices.

\section{Introduction}

Previous studies have demonstrated that the location of lesions in ischemic stroke patients is associated with the etiology, clinical symptoms, curative effect and prognosis of ischemic stroke (1-6). Magnetic resonance diffusion-weighted imaging (DWI), which is based on the diffusion of water molecules through a biological tissue, is widely used as a clinical tool to assess the extent of acute and hyperacute cerebral infarction (7). In conventional diffusion-weighted sequences, including DWI and apparent diffusion coefficient (ADC) maps, water molecule diffusion is assumed so that diffusion appears to be a free and nonrestricted environment within a Gaussian distribution of diffusion displacement $(8,9)$. However, the complex structure of different brain tissues, which consist of various distributions of cytons and fibers, can cause the diffusion displacement probability distribution to deviate substantially from a Gaussian form (9). Therefore, these simple models may not always detect the true diffusion of water molecule physiologically and pathologically, and the spatial and signal resolution of conventional diffusion-weighted sequences is not high enough to clearly distinguish between different brain regions (7-9). Diffusional kurtosis imaging (DKI) is a new type of diffusion-weighted sequence which measures the non-Gaussianity of water diffusion and may facilitate the characterization of different brain regions, including the corpus callosum, corona radiata, thalamus, subcortical white matter and cerebral cortex (9-11). Previous studies have demonstrated that DKI of ischemic stroke exhibited distinct abnormalities that are not detected by conventional DWI or ADC maps (12-14). Furthermore, animal and clinical studies have demonstrated the advantage of DKI to stratify heterogeneously damaged lesions (14-16). 
Previous studies on DKI of ischemic stroke have only concentrated on white matter and gray matter lesions and have predominantly focused on the comparison of DKI with conventional DWI/DTI (12-16). Furthermore, DKI has multiple parameters, including mean kurtosis (MK), axial kurtosis (Ka) and radial kurtosis (Kr), and, to the best of our knowledge, the diagnostic value of each of these parameters for cerebral infarction in various brain regions has not been reported. The present analysis differed from previous investigations into DKI and stroke as it was postulated that the parameters of DKI were capable of distinguishing between the differences in water molecule diffusion in different brain regions under the conditions of acute infarction.

The present study aimed to identify the optimal DKI parameter for accurately locating ischemic lesions in various brain regions. The diagnostic value of the three DKI parameters for acute cerebral infarction were systematically evaluated in various regions of the brain, including the corpus callosum, corona radiata, thalamus, subcortical white matter and cerebral cortex, which represented single diffusion orientation fibers, crossing fibers, and a mixture of cytons and fibers, arcuate fibers and cytons, respectively. The theory and methodology of the present study may act as the basis for further research on DKI in other domains.

\section{Materials and methods}

Subjects. A total of 28 patients with acute cerebral infarction in various regions of the brain were recruited for the present prospective study. All patients underwent magnetic resonance imaging (MRI) scans within $72 \mathrm{~h}$ (range, 0.5-72 h; mean, $41.6 \pm 24.5 \mathrm{~h}$ ) of stroke onset, and were followed-up by MRI or computed tomography imaging after 30 days (range, 32-86 days) to confirm the final permanent infarction regions. Stroke duration was determined based on onset time and 13 cases of multiple cerebral infarction combined with ADC values as assistant analysis to confirm the responsible foci. Only patients with acute cerebral infarction were included in the present study. Patients with original or secondary cerebral hemorrhage, cerebral tumors, degenerative brain disease, craniocerebral trauma, post craniocerebral operation, dyspnea, coma and contraindications to MRI were excluded from the present study. Institutional Review Board approval was obtained for all experiments. All participants provided written informed consent.

Imaging acquisition and processing. All patients underwent cranial DKI and conventional MRI, including T1WI, T2WI, fluid attenuation inversion recovery, DWI and ADC maps, using a GE 1.5T HDx Echo Speed Plus MRI scanner and 8-channel head coil (GE Healthcare Life Sciences, Chalfont, UK). DKI data were acquired using an echo planar imaging diffusion sequence with the following parameters: Repetition time/echo time, 5,000/95.6 msec; field of view, $240 \times 240 \mathrm{~mm}^{2}$; matrix, 128x128; number of excitations, 2; oblique axial slices [number/thickness $(\mathrm{mm}) / \mathrm{gap}(\mathrm{mm})], 18 / 5 / 0$; and 15 gradient encoding directions with three $\mathrm{b}$ values $\left(0,1000,2000 \mathrm{~s} / \mathrm{mm}^{2}\right)$ for each direction in a total time of 6 min and $45 \mathrm{sec}$. MK, Ka and $\mathrm{Kr}$ were derived using the DKI Functool 9 software on the GE ADW4.6 workstation (GE Healthcare Life Sciences).
DKI software is a research tool in the Functool environment developed by the GE Applied Science Lab. It fits all DWIs and minimally-diffusion-weighted image $\left(\mathrm{b}_{0}\right.$ image) to the DKI model described by the following equation:

$\ln \left[S(n, b) / S_{0}\right]=-b \sum_{i=1}^{3} \sum_{j=1}^{3} n_{i} n_{j} D_{i j}+\frac{1}{6} b^{2} \bar{D}^{2} \sum_{i=1}^{3} \sum_{j=1}^{3} \sum_{k=1}^{3} \sum_{l=1}^{3} n_{i} n_{j} n_{k} n_{l} W_{i j k l}$,

where $S(n, b)$ is the diffusion signal intensity for diffusion weighting (b) and diffusion encoding direction (n), $S_{0}$ is the signal intensity for $\mathrm{b}_{0}, \mathrm{D}_{\mathrm{ij}}$ and $\mathrm{W}_{\mathrm{ijkl}}$ are the components of the diffusion and kurtosis tensor, respectively. Following estimation of the tensors, FA, MD, Da, Dr, MK, Ka and Kr were derived.

Imaging analysis. Regions of interest (ROIs) (area, 6-9 $\mathrm{mm}^{2}$ ) were outlined within the most severe areas of each lesion in the various brain regions according to each independent parametric map. ROIs of the same size were located on a corresponding area of the normal-appearing contralateral hemisphere as controls. Control ROIs were outlined on the adjacent area of normal-appearing regions in cases with corpus callosum infarction. Multiple DKI parameters for all ROIs, including MK, $\mathrm{Kr}$ and $\mathrm{Ka}$, were measured by DKI software on the GE ADW4.6 workstation. Relative MK (rMK), relative $\mathrm{Ka}(\mathrm{rKa})$ and relative $\mathrm{Kr}(\mathrm{rKr})$ levels of ischemic lesions were calculated using the formula: Relative DKI $=\mathrm{DKI}_{\text {ischemic }} / \mathrm{DKI}$ contralateral.

Diagnosis of ischemic lesions and image analyses were conducted by two radiologists as single-blind studies. A third radiologist was consulted when there were differences in opinion between the two primary radiologists.

Statistical analysis. Major assessment indices of the diagnostic value of relative DKI parameters, rMK, rKa and $\mathrm{rKr}$, were analyzed by independent sample t-test. Minor assessment indices, including the homogeneity of the background image and contrast of adjacent structures, were analyzed by Moses test of extreme reactions and independent sample t-test, respectively. Statistical analyses were performed using SPSS 19.0 software (SPSS, Inc., Chicago, IL, USA). P<0.05 was considered to indicate a statistically significant difference.

\section{Results}

Patient characteristics. A total of 28 acute ischemic stroke patients were enrolled in the present study, including 15 males and 13 females aged 58.7 \pm 9.9 years old (range, $42-78$ years). Based on the brain region involved in the $\mathrm{MK}, \mathrm{Ka}$ and $\mathrm{Kr}$ maps, 112 groups of ROIs were outlined in the following regions: Corpus callosum $(n=17)$; corona radiata $(n=26)$; thalamus $(n=21)$; subcortical white matter $(n=24)$; and cerebral cortex $(n=24)$. As controls, ROIs were outlined in the corresponding areas of the normal-appearing contralateral hemisphere (95 groups with cerebral hemisphere infarction) or adjacent areas (17 groups with corpus callosum infarction).

Parametric maps of DKI for acute cerebral infarction in various brain regions. Parametric maps of DKI, including $\mathrm{MK}, \mathrm{Ka}$ and $\mathrm{Kr}$ maps, for acute cerebral infarction in different regions are presented in Fig. 1. The background of the Ka 
Table I. Multiparameter analysis of DKI (including MK, Ka and Kr values) for normal-appearing areas in various brain regions.

\begin{tabular}{|c|c|c|c|c|c|c|c|c|c|c|}
\hline \multirow[b]{2}{*}{ Parameter } & \multicolumn{2}{|c|}{ Corpus callosum } & \multicolumn{2}{|c|}{ Corona radiata } & \multicolumn{2}{|c|}{ Thalamus } & \multicolumn{2}{|c|}{$\begin{array}{l}\text { Subcortical } \\
\text { white matter }\end{array}$} & \multicolumn{2}{|c|}{ Cerebral cortex } \\
\hline & mean $\pm \mathrm{SD}$ & $\mathrm{CV}(\%)^{\mathrm{a}}$ & mean $\pm \mathrm{SD}$ & $\mathrm{CV}(\%)^{\mathrm{a}}$ & mean $\pm \mathrm{SD}$ & $\mathrm{CV}(\%)^{\mathrm{a}}$ & mean $\pm \mathrm{SD}$ & $\mathrm{CV}(\%)^{\mathrm{a}}$ & mean $\pm \mathrm{SD}$ & $\mathrm{CV}(\%)^{\mathrm{a}}$ \\
\hline MK & $1.00 \pm 0.07$ & 7.00 & $0.98 \pm 0.07$ & 7.14 & $0.88 \pm 0.07$ & 7.95 & $0.92 \pm 0.06$ & 6.52 & $0.83 \pm 0.04$ & 4.82 \\
\hline $\mathrm{Ka}$ & $0.73 \pm 0.03$ & 4.10 & $0.71 \pm 0.03$ & 4.23 & $0.68 \pm 0.03$ & 4.41 & $0.69 \pm 0.03$ & 4.35 & $0.68 \pm 0.03$ & 4.41 \\
\hline $\mathrm{Kr}$ & $1.18 \pm 0.14$ & 11.86 & $1.04 \pm 0.09$ & 8.65 & $0.94 \pm 0.09$ & 9.57 & $0.94 \pm 0.10$ & 10.64 & $0.89 \pm 0.05$ & 5.62 \\
\hline P-value ${ }^{b}$ & $<0.05$ & & $<0.05$ & & $<0.05$ & & $<0.05$ & & $<0.05$ & \\
\hline
\end{tabular}

${ }^{\mathrm{a}} \mathrm{CV}=(\mathrm{mean} / \mathrm{SD}) \times 100 \%$. ${ }^{\mathrm{b}} \mathrm{P}$-value of multiparameters of DKI value (including $\mathrm{MK}, \mathrm{Ka}$ and $\mathrm{Kr}$ values) in each brain region, calculated using Moses test of extreme reactions. DKI, diffusional kurtosis imaging; MK, mean kurtosis; Ka, axial kurtosis; Kr, radial kurtosis; CV, variation coefficient; SD, standard deviation.
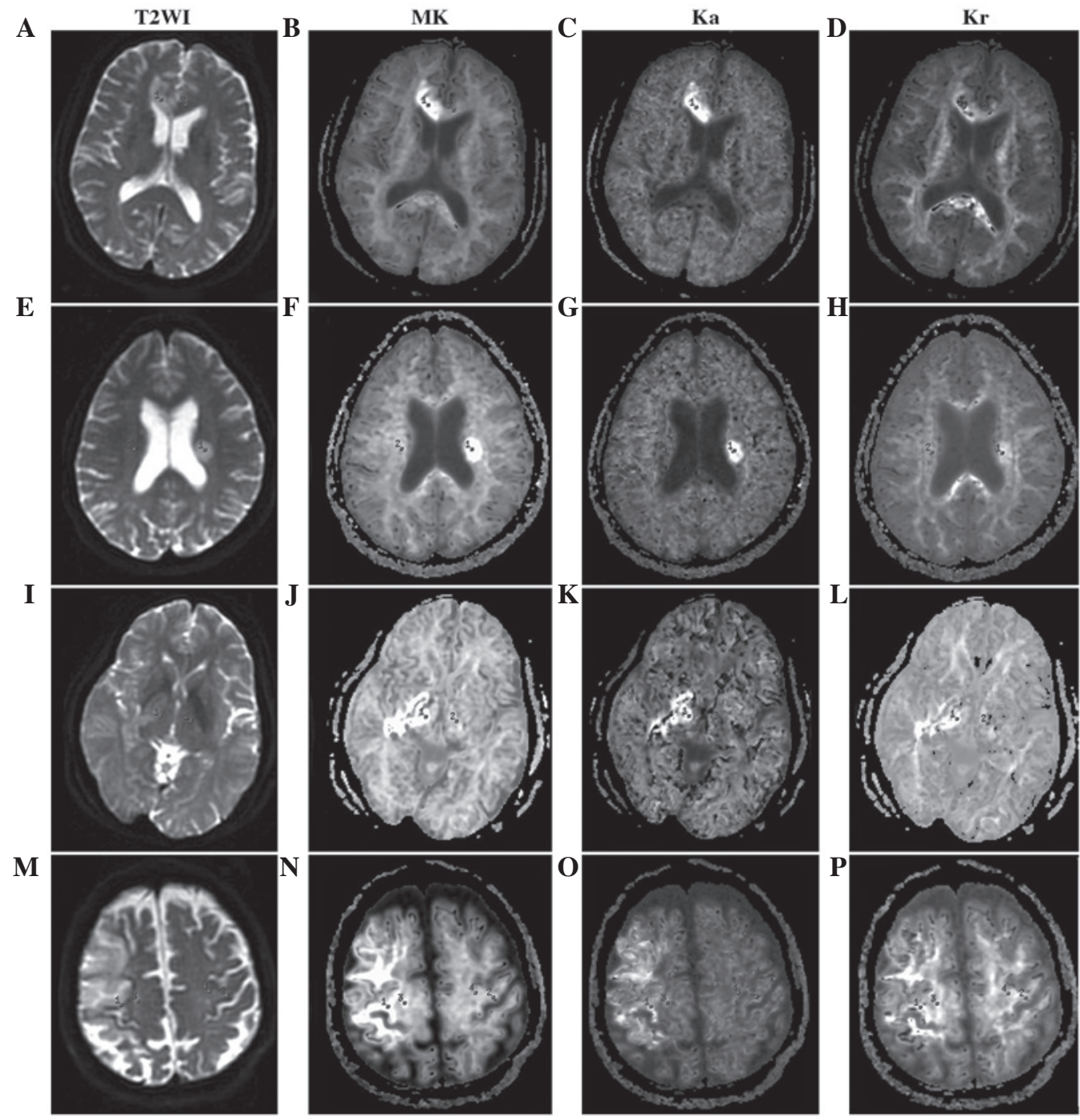

Figure 1. Parametric maps of DKI, including MK, Ka and Kr maps, for acute cerebral infarction in various regions of the brain. T2WI and parametric maps from the same anatomical slice of acute cerebral infarction for the (A-D) corpus callosum, (E-H) corona radiata, (I-L) thalamus and (M-P) subcortical white matter and cerebral cortexes. (A-L) ROIs for ischemic lesions are indicated by ROI 1, and ROIs for corresponding areas of the normal-appearing contralateral hemisphere are indicated by ROI 2. (M-P) ROIs for lesion and control in cerebral cortexes are indicated by ROI 1 and ROI 2, respectively, and ROIs for lesion and control in subcortical white matter are indicated by ROI 3 and ROI 4, respectively. DKI, diffusional kurtosis imaging; MK, mean kurtosis; Ka, axial kurtosis; Kr, radial kurtosis; ROI, region of interest.

maps was relatively homogeneous, and ischemic lesions were detected in the corpus callosum and corona radiate. However, due to the lack of contrast of adjacent structures, ischemic lesions in the thalamus, subcortical white matter and cerebral 
cortices were less clear on Ka maps, as compared with the other parametric maps. The background of the Kr maps was heterogeneous, particularly in the corpus callosum, corona radiate and subcortical white matter, indicating that this map was unable to detect ischemic lesions in these regions. For the MK maps, the signal strength of ischemic lesions, the homogeneity of background image and contrast of adjacent structures lay between the $\mathrm{Kr}$ and $\mathrm{Ka}$ maps. Ischemic lesions in the thalamus, subcortical white matter and cerebral cortices were most detectable on MK maps.

Parameters of DKI for acute cerebral infarction and normal-appearing areas in various brain regions. DKI parameters, including $\mathrm{MK}, \mathrm{Ka}$ and $\mathrm{Kr}$ values, of normal-appearing regions are shown in Table I and Fig. 2. The dispersions of MK, $\mathrm{Ka}$ and $\mathrm{Kr}$ values of the normal-appearing areas in each brain region were statistically different $(\mathrm{P}<0.05)$, as determined by Moses test of extreme reactions and variation coefficient (Table I), indicating that the homogeneity of the background images of parametric maps was different. The MK, $\mathrm{Ka}$ and $\mathrm{Kr}$ values of the normal-appearing area in different brain regions were inconsistent, as determined by independent sample t-test, which indicated that the contrast of adjacent structures of parametric maps was different (Fig. 2). MK, $\mathrm{Ka}$ and $\mathrm{Kr}$ values were significantly increased in the ischemic region, as compared with the corresponding areas of the normal-appearing region (P<0.05; Fig. 3).

Relative DKI parameters for acute cerebral infarction in different brain regions. Relative DKI rMK, rKa and rKr parameters are shown in Fig. 4. For ischemic regions in the corpus callosum and corona radiate, rKa was significantly increased, as compared with other DKI parameters $(\mathrm{P}<0.05)$ determined by independent sample t-test. However, for ischemic regions in the thalamus, subcortical white matter and cerebral cortex, the increase of rMK, rKa and $\mathrm{rKr}$ values not significant, as compared with the other DKI parameters.

\section{Discussion}

Homogeneity of an image background is associated with the detection of lesions, and the contrast of adjacent structures contribute to the accurate positioning of lesions (9). Therefore, it is necessary to study these two assessment indices in different normal-appearing brain regions prior to lesion analysis.

To the best of our knowledge, the present study was the first to report homogeneity of the background images of different parametric maps of DKI, and use variation coefficient and Moses test of extreme reactions to analyze the dispersion of DKI parameters, which reflect the homogeneity of parametric image backgrounds. The results of the present study demonstrated that the variation coefficient of DKI parameters in each brain region were $\mathrm{Kr}$, MK and $\mathrm{Ka}$ value from the maximum to the minimum, respectively, and the differences were statistically significant $(\mathrm{P}<0.05)$, as determined by Moses test of extreme reactions.

Previous studies have demonstrated that the MK values of different brain tissues are distinct $(9,11)$; however, the contrast of adjacent structures on different parametric maps of DKI has not been reported. The results of the present
A

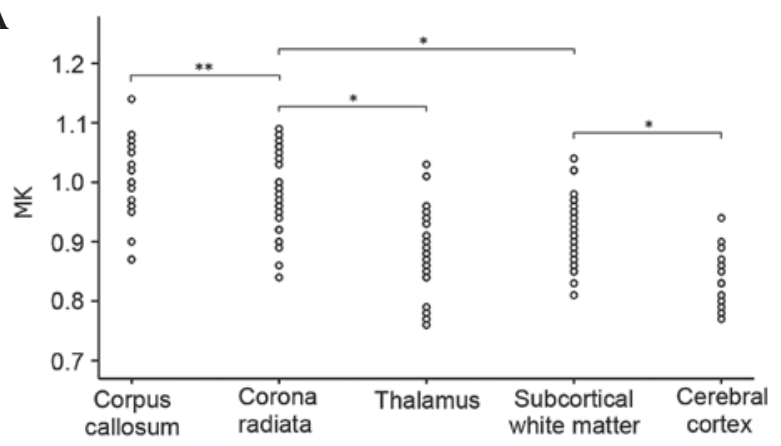

B

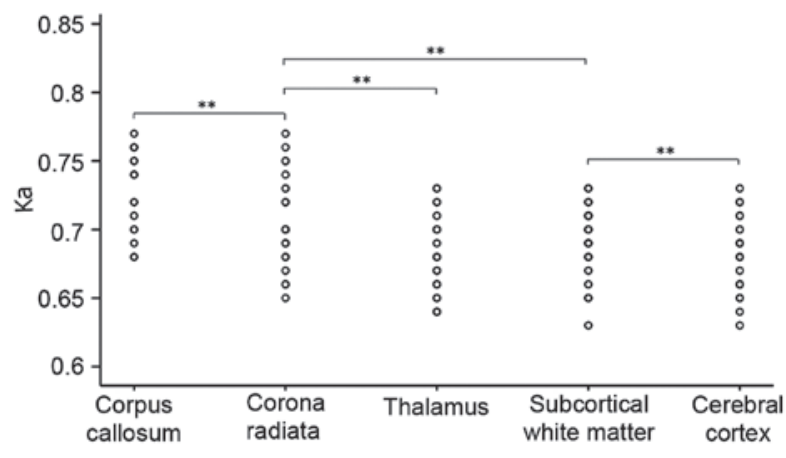

C

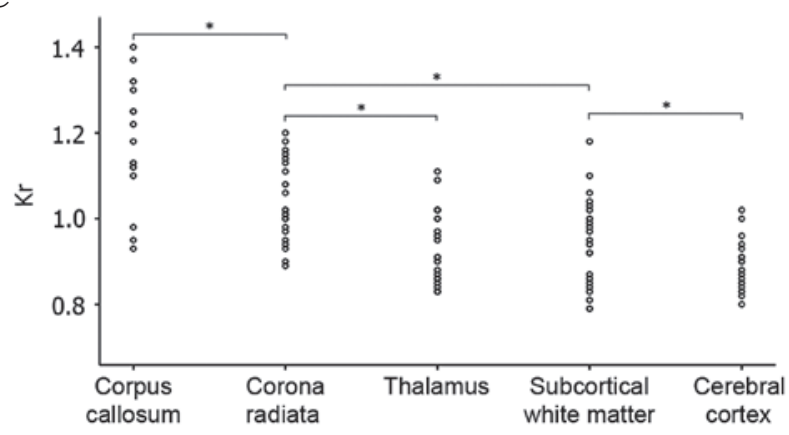

Figure 2. Scatterplots for multiparameters of DKI, including (A) MK, (B) Ka and $(\mathrm{C}) \mathrm{Kr}$ values, of normal-appearing areas in various regions of the brain. $\mathrm{MK}, \mathrm{Ka}$ and $\mathrm{Kr}$ values of the normal-appearing region in different brain region were inconsistent, as determined by independent sample t-test. ${ }^{*} \mathrm{P}<0.05 ;{ }^{* *} \mathrm{P}>0.05$. DKI, diffusional kurtosis imaging; MK, mean kurtosis; $\mathrm{Ka}$, axial kurtosis; Kr, radial kurtosis.

study demonstrated that the Ka values of adjacent structures in normal-appearing regions were not significantly different; whereas the differences in $\mathrm{Kr}$ values were statistically significant $(\mathrm{P}<0.05)$. Although no significant differences in the MK values of the corpus callosum and corona radiata were detected, the following brain regions exhibited significant differences in MK values: Corona radiata vs. thalamus; corona radiata vs. subcortical white matter; and subcortical white matter vs. cerebral cortices $(\mathrm{P}<0.05)$.

Corresponding to the statistical analyses of MK, Ka and $\mathrm{Kr}$ values of the normal-appearing region in various brain regions, it was observed that the background of Ka maps was relatively homogeneous with less contrast of adjacent structures; the background of $\mathrm{Kr}$ maps was heterogeneous with strong contrast; whereas, for MK maps, the homogeneity of the image background and contrast of adjacent structures was between that of the $\mathrm{Kr}$ and $\mathrm{Ka}$ maps. 
A

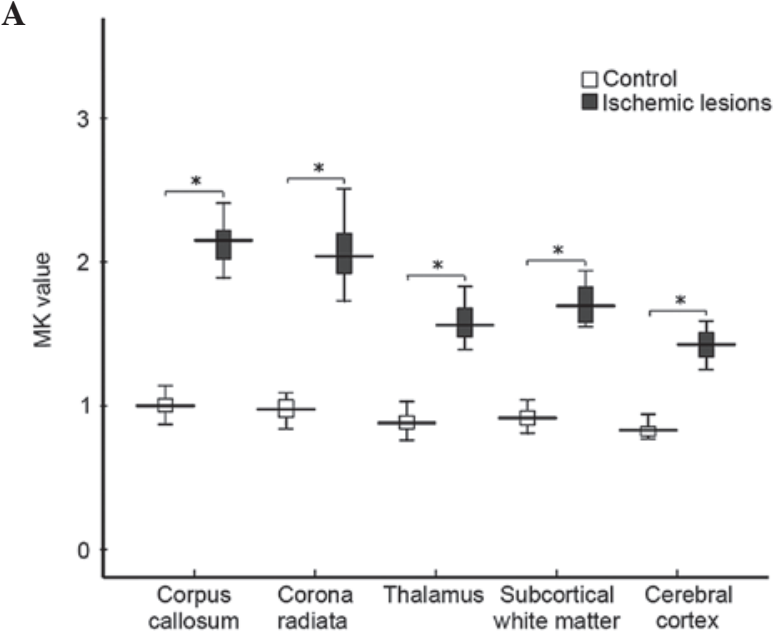

B

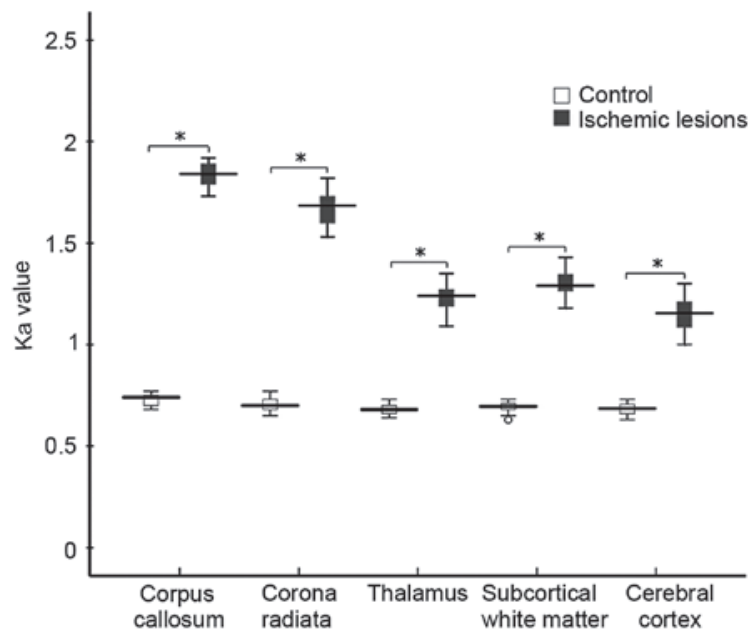

C

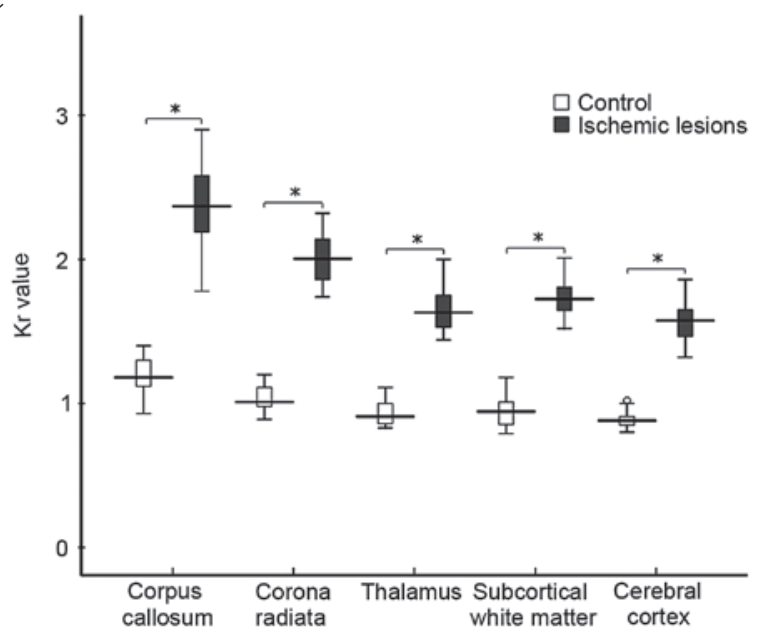

Figure 3. Multiparameters of DKI, including (A) MK, (B) $\mathrm{Ka}$ and (C) $\mathrm{Kr}$ values) for the diagnosis of acute cerebral infarction in various regions of the brain. * $\mathrm{P}<0.05$. DKI, diffusional kurtosis imaging; MK, mean kurtosis; $\mathrm{Ka}$, axial kurtosis; Kr, radial kurtosis.

Although the present study demonstrated that the absolute MK, Ka and Kr DKI values altered in the normal-appearing region of different brain regions, the practical implications are limited. Percent change is the most common method to evaluate the DKI value of lesions; however, the calculation is

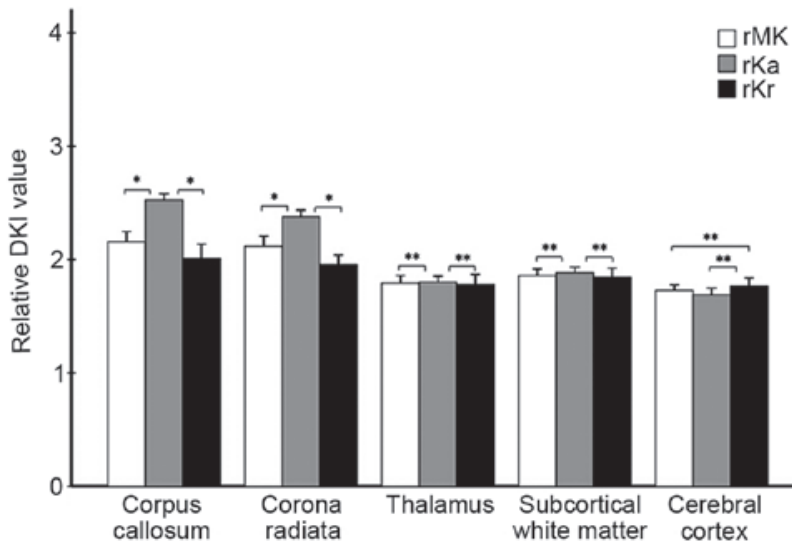

Figure 4. Relative values of the parameters of DKI, including rMK, rKa and $\mathrm{rKr}$, for the diagnosis of acute cerebral infarction in various regions of the brain. ${ }^{*} \mathrm{P}<0.05 ;{ }^{* *} \mathrm{P}>0.05$. DKI, diffusional kurtosis imaging; rMK, relative mean kurtosis; rKa, relative axial kurtosis; rKr, relative radial kurtosis.

complicated and is thus not appropriate for clinical application (12-14). Calculating the relative DKI value, including $\mathrm{rMK}, \mathrm{rKa}$ and $\mathrm{rKr}$ values, and the ratio of lesional DKI value to corresponding area of the normal-appearing region is easily accessible and may reduce the instability of absolute DKI value. The results of the present study demonstrated that the $\mathrm{MK}, \mathrm{Ka}$ and $\mathrm{Kr}$ values were significantly increased in ischemic lesions, as compared with the normal-appearing region in every brain region; however, the increases of rMK, rKa and $\mathrm{rKr}$ values were different in the various brain regions. These results suggested that the $\mathrm{rMK}$, $\mathrm{rKa}$ and $\mathrm{rKr}$ values displayed the variation of $\mathrm{MK}, \mathrm{Ka}$ and $\mathrm{Kr}$ values and were more applicable to quantitative analysis for lesions.

To evaluate the diagnostic value of the multiple parameters of DKI for acute cerebral infarction in different regions of the brain, a set of assessment indices was established. Major assessment indices included rMK, rKa and $\mathrm{rKr}$, which facilitated the evaluation of the diagnostic value of multiparameter DKI objectively, based on quantitative analysis. Minor assessment indices included the homogeneity of background and the contrast of adjacent structures. Among these assessment indices, MK, $\mathrm{Ka}$ and $\mathrm{Kr}$ exhibited varying diagnostic values for acute cerebral infarction in different brain regions.

For ischemic lesions in highly anisotropic brain regions, such as the corpus callosum and corona radiata, significant increases in rKa were detected, as compared with the other DKI parameters $(\mathrm{P}<0.05)$. These results are consistent with previous studies, which have suggested that axonal varicosities and endoplasmic reticulum alterations are the potential sources of higher diffusion restriction of $\mathrm{Ka}$ in white matter infarction (12-14). Furthermore, due to the relatively homogeneous background, Ka maps enabled an improved view of ischemic lesions in the corpus callosum and corona radiata, as compared with the other DKI parametric maps.

For ischemic lesions in low anisotropic or nearly isotropic brain regions such as the thalamus, subcortical white matter and cerebral cortices, the increases in $\mathrm{rMK}, \mathrm{rKa}$ and $\mathrm{rKr}$ were not significant. Therefore, minor assessment indices should be included as supplementary criteria in these brain regions. The structures of these brain regions were relatively 
complicated and Ka was not competent enough to enable accurate positioning of the ischemic lesions due to the lack of tissue contrast. Although $\mathrm{Kr}$ exhibited favorable tissue contrast, the background was heterogeneous which may interfere with the detection of lesions. Therefore, MK was the superior assessment parameter for ischemic lesions in these regions, as the homogeneity of the background was optimally balanced with the contrast of adjacent structures. Furthermore, MK is considered to be the only diffusional sequence that can be applied to nearly isotropic brain regions, such as the cerebral cortices and thalamus $(14,17-22)$.

Accurate localization of cerebral infarction is associated with clinical symptoms, curative effect and the prognosis of ischemic stroke (1-4). In the present study the diagnostic value of DKI parameters was evaluated for acute cerebral infarction in various brain regions. The results demonstrated that $\mathrm{MK}, \mathrm{Ka}$ and $\mathrm{Kr}$ exhibited different diagnostic values for acute cerebral infarction in the different brain regions. It was demonstrated that $\mathrm{Ka}$ is better suited to the diagnosis of acute ischemic lesions containing single diffusion orientation fibers and crossing fibers, such as in the corpus callosum and corona radiata. MK may be appropriate for the diagnosis of acute ischemic lesions containing a mixture of cytons and fibers, arcuate fibers, and cytons, such as in the thalamus, subcortical white matter and cerebral cortices, respectively.

In conclusion, the results of the present study demonstrated that DKI is capable of distinguishing between the differences in water molecule diffusion in various brain regions under the conditions of acute infarction. The selective utilization of DKI parameters will help make accurate positioning of acute cerebral infarction, and provide a promising new method for the diagnosis of ischemic stroke.

\section{Acknowledgements}

The present study was supported by the National Natural Science Foundation of China (grant no. 81471730), the Science and Technology Planning Project of Guangdong Province, China (grant no. 2010B031600276), and the National High Technology Research and Development Program of China (grant no. 2014AA021101).

\section{References}

1. Galovic M, Leisi N, Müller M, Weber J, Abela E, Kägi G and Weder B: Lesion location predicts transient and extended risk of aspiration after supratentorial ischemic stroke. Stroke 44: 2760-2767, 2013

2. Tang WK, Chen YK, Liang HJ, Chu WC, Mok VC, Ungvari GS and Wong KS: Location of infarcts and apathy in ischemic stroke. Cerebrovasc Dis 35: 566-571, 2013.

3. Phan TG, Demchuk A, Srikanth V, Silver B, Patel SC, Barber PA, Levine SR and Hill MD: Proof of concept study: Relating infarct location to stroke disability in the NINDS rt-PA Trial. Cerebrovasc Dis 35: 560-565, 2013.

4. Long B, Spencer-Smith MM, Jacobs R, Mackay M, Leventer R, Barnes $C$ and Anderson V: Executive function following child stroke: The impact of lesion location. J Child Neurol 26: 279-287, 2011.
5. Sillanpää N, Saarinen JT, Rusanen H, Elovaara I, Dastidar P and Soimakallio S: Location of the clot and outcome of perfusion defects in acute anterior circulation stroke treated with intravenous thrombolysis. AJNR Am J Neuroradiol 34: 100-106, 2013.

6. Jickling GC, Stamova B, Ander BP, Zhan X, Liu D, Sison SM, Verro P and Sharp FR: Prediction of cardioembolic, arterial, and lacunar causes of cryptogenic stroke by gene expression and infarct location. Stroke 43: 2036-2041, 2012.

7. Wu RH, Bruening R, Haberl R, Deimling M, Porn U and Reiser M: Early detection of human brain infarction by using diffusion weighted imaging. Zhonghua Nei Ke Za Zhi 37: 116-118, 1998.

8. Yan G, Dai Z, Xuan Y and Wu R: Early metabolic changes following ischemia onset in rats: An in vivo diffusion-weighted imaging and $1 \mathrm{H}$-magnetic resonance spectroscopy study at $7.0 \mathrm{~T}$. Mol Med Rep 11: 4109-4114, 2015.

9. Chen Y, Zhao X, Ni H, Feng J, Ding H, Qi H, Wan B and Ming D: Parametric mapping of brain tissues from diffusion kurtosis tensor. Comput Math Methods Med 2012: 820847, 2012.

10. Hori M, Tsutsumi S, Yasumoto Y, Ito M, Suzuki M, Tanaka FS, Kyogoku S, Nakamura M, Tabuchi T, Fukunaga I, et al: Cervical spondylosis: Evaluation of microstructural changes in spinal cord white matter and gray matter by diffusional kurtosis imaging. Magn Reson Imaging 32: 428-432, 2014.

11. Lätt J, Nilsson M, Wirestam R, Ståhlberg F, Karlsson N, Johansson M, Sundgren PC and van Westen D: Regional values of diffusional kurtosis estimates in the healthy brain. J Magn Reson Imaging 37: 610-618, 2013.

12. Jensen JH, Falangola MF, Hu C, Tabesh A, Rapalino O, Lo C and Helpern JA: Preliminary observations of increased diffusional kurtosis in human brain following recent cerebral infarction. NMR Biomed 24: 452-457, 2011.

13. Hui ES, Fieremans E, Jensen JH, Tabesh A, Feng W, Bonilha L, Spampinato MV, Adams R and Helpern JA: Stroke assessment with diffusional kurtosis imaging. Stroke 43: 2968-2973, 2012.

14. Hui ES, Du F, Huang S, Shen Q and Duong TQ: Spatiotemporal dynamics of diffusional kurtosis, mean diffusivity, and perfusion changes in experimental stroke. Brain Res 1451: 100-109, 2012.

15. Cheung JS, Wang E, Lo EH and Sun PZ: Stratification of heterogeneous diffusion MRI ischemic lesion with kurtosis imaging: Evaluation of mean diffusion and kurtosis MRI mismatch in an animal model of transient focal ischemia. Stroke 43: 2252-2254, 2012.

16. Sun PZ, Wang Y, Mandeville E, Chan ST, Lo EH and Ji X: Validation of fast diffusion kurtosis MRI for imaging acute ischemia in a rodent model of stroke. NMR Biomed 27: 1413-1418, 2014.

17. Lazar M, Jensen JH, Xuan L and Helpern JA: Estimation of the orientation distribution function from diffusional kurtosis imaging. Magn Reson Med 60: 774-781, 2008.

18. Grinberg F, Farrher E, Ciobanu L, Geffroy F, Le Bihan D and Shah NJ: Non-Gaussian diffusion imaging for enhanced contrast of brain tissue affected by ischemic stroke. PLoS One 9: e89225, 2014.

19. Filli L, Wurnig M, Nanz D, Luechinger R, Kenkel D and Boss A: whole-body diffusion kurtosis imaging: Initial experience on Non-Gaussian diffusion in various organs. Invest Radiol 49: 773-778, 2014.

20. Gao Y, Zhang Y, Wong CS, Wu PM, Zhang Z, Gao J, Qiu D and Huang B: Diffusion abnormalities in temporal lobes of children with temporal lobe epilepsy: A preliminary diffusional kurtosis imaging study and comparison with diffusion tensor imaging. NMR Biomed 25: 1369-1377, 2012.

21. Helpern JA, Adisetiyo V, Falangola MF, Hu C, Di Martino A, Williams K, Castellanos FX and Jensen JH: Preliminary evidence of altered gray and white matter microstructural development in the frontal lobe of adolescents with attention-deficit hyperactivity disorder: A diffusional kurtosis imaging study. J Magn Reson Imaging 33: 17-23, 2011.

22. Falangola MF, Jensen JH, Babb JS, Hu C, Castellanos FX, Di Martino A, Ferris SH and Helpern JA: Age-related non-Gaussian diffusion patterns in the prefrontal brain. J Magn Reson Imaging 28: 1345-1350, 2008. 